\title{
Dimensionamento de recursos humanos em serviços de alimentação e nutrição de hospitais públicos e privados*
}

\author{
Rita de Cássia Lusia dos Santos** \\ Rosa Wanda Diez-Garcia***
}

Sumário: 1. Introdução; 2. Material e métodos; 3. Resultados; 4. Discussão; 5. Conclusão.

Summary: 1. Introduction; 2. Material and methods; 3. Results; 4. Discussion; 5. Conclusion.

Palavras-chave: nutrição hospitalar; recursos humanos; serviços de alimentação hospitalar.

KEY WORDs: hospital nutrition; human resources; profile of dietitian.

O objetivo deste trabalho foi caracterizar e comparar o dimensionamento de recursos humanos dos Serviços de Alimentação e Nutrição Hospitalar (SANH) e a qualificação do corpo técnico de nutricionistas da rede hospitalar pública e privada. Foram estudados 27 hospitais, 17 de Campinas e 10 de Ribeirão Preto, por meio de um questionário estruturado aplicado aos coordenadores do SANH, que abordou: condições de trabalho, pela relação entre número de leitos por nutricionista e por profissionais do SANH, e pelo número de refeições produzidas por leito, por nutricionistas e por funcionários do SANH; a formação acadêmica; e a experiência profissional dos nutricionistas. Foram encontradas diferenças significativas na relação número de leitos/nutricionista entre hospitais públicos $(38,2$ dp 11,4) e privados $(94,6$ dp 46,4) e de funcionários do

\footnotetext{
* Artigo recebido em dez. 2010 e aceito em jun. 2011.

** Nutricionista do Departamento de Clínica Médica, Divisão de Nutrição e Metabolismo da Faculdade de Medicina de Ribeirão Preto (FMRP) da Universidade de São Paulo (USP). Endereço: rua Vereador Horácio Arantes Silva, 392 - Jardim Procópio - CEP14065-450, Ribeirão Preto, SP, Brasil. E-mail: ritaclsantos57@yahoo.com.br.

*** Professora doutora do curso de nutrição e metabolismo e do Laboratório de Práticas e Comportamento Alimentares (PrátiCA) da FMRP/USP. Endereço: rua Ottorino Rizzi, 461, Royal Park — Bonfim Paulista - CEP 14110-000, Ribeirão Preto, SP, Brasil. E-mail: wanda@fmrp.usp.br.
} 
SANH/nutricionista (11,4 dp 4,9 e 23,8 dp 13,3). A produção de refeições por leitos foi maior nos hospitais públicos $(\mathrm{p}=0,04)$. No setor público concentram-se nutricionistas com capacitação stricto sensu, mesmo que ainda em um número reduzido em relação ao encontrado no país; e, no privado, há mais profissionais com formação lato sensu. Uma ampla heterogeneidade no dimensionamento de recursos humanos do SANH em hospitais públicos e privados foi encontrada; contudo, o setor público apresentou condição mais favorável em alguns indicadores, mesmo estando aquém das necessidades, principalmente na área de atenção nutricional ao paciente hospitalizado. Os resultados apontam para a necessidade de se constituirem indicadores específicos de recursos humanos nos SANHs, para o desempenho de ações na área de produção de refeições e de cuidado nutricional ao paciente hospitalizado. Criar mecanismos de capacitação do nutricionista para as áreas de competência da nutrição hospitalar poderia contribuir com o aprimoramento do setor.

Human resources dimensioning in food and nutrition hospital services in public and private hospitals

The objective of this paper was to characterize and compare the human resources dimensioning in food and nutrition hospital services (FNHS) and the qualification of the dietitian technical personnel in public and private hospitals. We studied 27 hospitals, 17 from Campinas and 10 from Ribeirão Preto, through a structured questionnaire applied to coordinators of the FNHS. The questionnaires addressed working conditions, by the number of beds per dietitian and per FNHS workers and number of meals per beds, per dietitian and per FNHS workers; academic background; and professional experience of dietitians. We found significant differences in the number of beds per dietitian between public (38.2 SD 11.4) and private (94.6 SD 46.4) hospitals, and of FNHS workers per dietitian (11.4 SD 4.9 and 23.8 SD 13.3 for public and private hospitals respectively). The number of meals per bed was greater in public hospitals $(\mathrm{p}=0.04)$. Dietitians with stricto sensu post-graduation degrees are concentrated in public hospitals whereas the ones with lato sensu post-graduation degrees are present in larger numbers in private hospitals. The wide heterogeneity in the dimension of human resources of FNHS in public and private hospitals has been found yet. The public setor showed more favorable conditions hospitals had in some indicators, although short of the needs in the area mainly for nutritional care for hospitalized patients. The results point to the need to provide specific indicators of human resources in FNHS, for the performance of shares in the production of food and nutritional care to hospitalized patients. Build mechanisms for training of nutritionists for areas of competence of hospital food could contribute to improvement of the sector.

\section{Introdução}

A atenção nutricional hospitalar agrega ações relacionadas ao monitoramento do estado nutricional e tratamento dietético envolvidos no cuidado do pa- 
ciente hospitalizado (Vasconcelos, 2002; Conselho Federal de Nutricionistas, 2005) e ações de gestão de unidades de alimentação e nutrição, direcionadas para a produção da alimentação hospitalar (Conselho Federal de Nutricionistas, 2005). A área hospitalar demanda, portanto, nutricionistas habilitados em nutrição clínica e em gestão de unidades de alimentação e nutrição, pois compete a cada segmento um rol de responsabilidades específicas que, em conjunto, garantem a qualidade deste tipo de assistência.

Apesar da abrangência de suas responsabilidades profissionais, a definição do papel do nutricionista por parte da instituição hospitalar é ainda incipiente, com ações de diferentes graus de complexidade e heterogêneas entre hospitais e em setores de uma mesma instituição (Souza e Proença, 2004; Garcia, 2006). O impacto que a depleção do estado nutricional tem nos custos, no tempo de permanência na internação (McMahon, Decker, Ottery, 1998; Leandro-Merhi et al., 2006) e o papel da assistência nutricional na reabilitação do paciente internado (Oliveira et al., 2005) são suficientes para justificar a importância deste cuidado. As diferentes expectativas por parte dos hospitais em relação ao Serviço de Alimentação e Nutrição Hospitalar (SANH) expressam a falta de conhecimento sobre o papel deste profissional e do impacto que o cuidado nutricional tem no paciente hospitalizado (Garcia, 2006; LeandroMerhi, Garcia e Aquino, 2007). As relações de poder na estrutura organizacional das instituições hospitalares concentradas no médico contribuem para limitar os campos de atuação de outros profissionais da área da saúde (Farias e Vaitsman, 2002; Bosi, 1996).

O aspecto organizacional do setor hospitalar influencia a atuação de profissionais da área da saúde, de acordo com estudo que analisou a relação entre o número de funcionários e o número de leitos de hospitais públicos e privados do estado de São Paulo, indicador de planejamento dos recursos humanos e de custos hospitalares (Zuchhi e Bittar, 2002). A construção de indicadores na área de assistência nutricional hospitalar poderia contribuir para a qualidade do setor.

A falta de autonomia técnica, o grande número de leitos por nutricionistas e a falta de reconhecimento de suas competências prejudicam o desempenho de suas ações, particularmente na área de cuidado nutricional ao paciente hospitalizado (Bosi, 1996; Prado e Abreu, 1991). A capacitação é reconhecidamente importante no processo de qualificação e pode facilitar a inserção e o reconhecimento do profissional (Bosi, 1996). Segundo estudo nacional sobre o perfil do nutricionista, é ainda incipiente o número de profissionais com capacitação stricto e lato sensu (Conselho Federal de Nutricionistas, 2006).

Foi objetivo deste estudo caracterizar e comparar o dimensionamento de recursos humanos dos SANHs e analisar a qualificação de seu corpo técnico 
em hospitais públicos e privados de dois municípios considerados de referência para o setor da saúde.

\section{Material e métodos}

Foram estudados 27 hospitais de dois municípios do estado de São Paulo: 17 de Campinas, município de aproximadamente um milhão de habitantes; e 10 de Ribeirão Preto, com cerca de 550 mil habitantes. As cidades foram escolhidas por serem referência na área da saúde, concentrarem várias universidades e por serem polos tecnológico e econômico do Estado. As instituições foram caracterizadas por natureza jurídica, tipo e tamanho.

Foram critérios de exclusão, hospitais psiquiátricos, hospital dia, abrigo e casa de repouso, não haver nutricionista responsável pelo SANH, e a instituição não aceitar participar da pesquisa. Foi aplicado aos coordenadores do SANH, no próprio local de trabalho, um questionário estruturado, contendo questões abertas e fechadas, no qual foram abordadas: as condições de trabalho e a formação e a experiência profissional. Os dados gerais dos hospitais foram colhidos do sistema Datasus (Ministério da Saúde, 2007).

As condições de trabalho foram verificadas pelos seguintes indicadores: número de nutricionistas por hospital, número de leitos por nutricionistas, número de leitos por funcionários do $\mathrm{SANH}$, número de funcionários do $\mathrm{SANH}$ por nutricionistas e número de refeições produzidas por nutricionistas e por funcionários do SANH. Foi averiguado também o sistema de plantão no SANH (considerado por área - nutrição clínica e produção de refeições, com a presença física do nutricionista) e o regime de trabalho (em horas/dia de trabalho). A existência de computador e as atividades informatizadas no serviço foram verificadas como um indicador de infraestrutura do serviço.

A formação e a experiência profissional foram avaliadas considerandose o nível de capacitação do nutricionista (especialização, mestrado e doutorado), a afinidade entre a capacitação e a área de atuação (nutrição clínica, administração de unidades de alimentação e saúde pública), o tempo de graduado e a prática de frequentar cursos de atualização nos últimos dois anos, e a área de atuação no último emprego para conhecer a experiência profissional pregressa à atividade hospitalar.

Foi realizada estatística descritiva por segmento hospitalar público e privado e por município. Para as variáveis que representam contagens foram propostos modelos de Poisson com efeito aleatório para controlar a dispersão (Coelho-Barros et al., 2005). Para as demais comparações foram utilizados 
modelos de Anova (Montgomery, 2000). Os ajustes dos modelos foram feitos através do software SAS versão 9 (SAS, 2003).

\section{Resultados}

Os 27 hospitais estudados representam 82,5\% dos leitos de Campinas e 87,5\% dos leitos de Ribeirão Preto, segundo a disponibilidade de leitos descrita na base de dados Datasus, a qual inclui leitos psiquiátricos, excluídos deste estudo. A disponibilidade de leitos por mil habitantes no estado de São Paulo é de 2,29 leitos/1.000 habitantes (0,58 leito público e 1,71 leito privado/1.000 habitantes). A região metropolitana de Campinas tem maior proporção $(74,6 \%)$ de leitos privados (de 1,97 leito/1.000 habitantes, 1,47 é privado) (Ministério da Saúde, 2007).

Tabela 1

Características dos hospitais estudados, estado de São Paulo, Brasil, 2005

\begin{tabular}{|lcccc|}
\hline Características dos Hospitais & $\begin{array}{c}\text { Campinas } \\
(\mathrm{n}=17)\end{array}$ & $\begin{array}{c}\text { Ribeirão Preto } \\
(\mathrm{n}=10)\end{array}$ & $\begin{array}{c}\text { Total } \\
(\mathrm{n}=27)\end{array}$ & $\%$ \\
\hline $\begin{array}{l}\text { Natureza Jurídica } \\
\quad \text { Públicos ( } \mathrm{n}=5)\end{array}$ & & & & \\
Universitários & 2 & 2 & 4 & 14,8 \\
Não Universitários & 1 & 0 & 1 & 3,7 \\
$\quad$ Total Públicos & 3 & 2 & 5 & 18,5 \\
$\quad$ Privados ( $\mathrm{n=22)}$ & 1 & & & \\
Universitário & 7 & 4 & 11 & 4,4 \\
Empresa privada & 6 & 3 & 9 & 33,3 \\
Filantrópico & 14 & 8 & 22 & 81,5 \\
Total Privados & & & & \\
Tipo & 3 & 2 & 5 & 18,5 \\
Especializado & 14 & 8 & 22 & 81,5 \\
Tamal & & & & \\
Pequeno (até 50 leitos) & 2 & 1 & 3 & 11,1 \\
Médio (mais de 50 até 150) & 11 & 6 & 17 & 63,0 \\
Grande (mais de 150) & 4 & 3 & 7 & 25,9 \\
\hline
\end{tabular}


Por se tratar de um estudo em duas cidades com características similares em termos de equipamento de saúde, conforme era esperado, não foram observadas diferenças significantes nos indicadores de dimensionamento de recursos humanos de SANH entre os dois municípios estudados nem nos indicadores de nível de capacitação e áreas de capacitação e atuação. Tanto aqueles indicadores que tratam da relação com o número de leitos como os que se referem à produção de refeições apresentaram ampla margem de variação, indicando uma heterogeneidade na composição funcional dos SANHs entre os hospitais, independentemente do segmento analisado (tabela 2). Todavia, houve diferença estatística significante entre hospitais públicos e privados em relação ao número de leitos e número de funcionários do SANH por nutricionista.

Foi nas instituições públicas que se observou um maior número de refeições por leito $(\mathrm{p}<0,04)$, resultado obtido pelo número total de refeições produzidas dividida pelo número de leitos do hospital. Pode ter contribuído para a variação do número de refeições produzidas o fato de haver serviços que produzem refeições para outros segmentos, como para funcionários e mesmo para serviços externos, chegando a produzir um volume de refeições maior do que para os pacientes. Isso indica também o risco de deslocar o objetivo do serviço que, em vez de ter como prioridade o paciente hospitalizado, despende recursos humanos para produzir refeições para outros segmentos.

Foram investigados 74 nutricionistas, 44 pertencentes ao município de Campinas e 30 de Ribeirão Preto (tabela 3). Dois terços dos nutricionistas que trabalham nos hospitais têm especialização e $1 / 3$, apenas graduação. Ainda é pouco expressivo o número de profissionais com pós-graduação stricto sensu e aqueles que têm esta titulação concentram-se no setor público. No setor privado há mais nutricionistas com especialização do que nos hospitais públicos. A maioria $(66,7 \%)$ fez algum tipo de capacitação em nutrição clínica ou área afim. É no setor privado que o nutricionista acumula a função de gerência, clínica e produção $(57,6 \%)$, principalmente em Ribeirão Preto (88,9\%). Tal condição põe em pauta a discussão sobre a viabilidade de se exercer habilidades nestas áreas simultaneamente e, na prática, quais ações são priorizadas e quais deixam de ser desenvolvidas. Mesmo não sendo observada diferença estatística no número de nutricionistas que atuam na área de nutrição clínica, nos hospitais públicos e privados, $58,5 \%$ dos hospitais públicos e $21,2 \%$ dos privados têm nutricionistas específicos para esta área. 


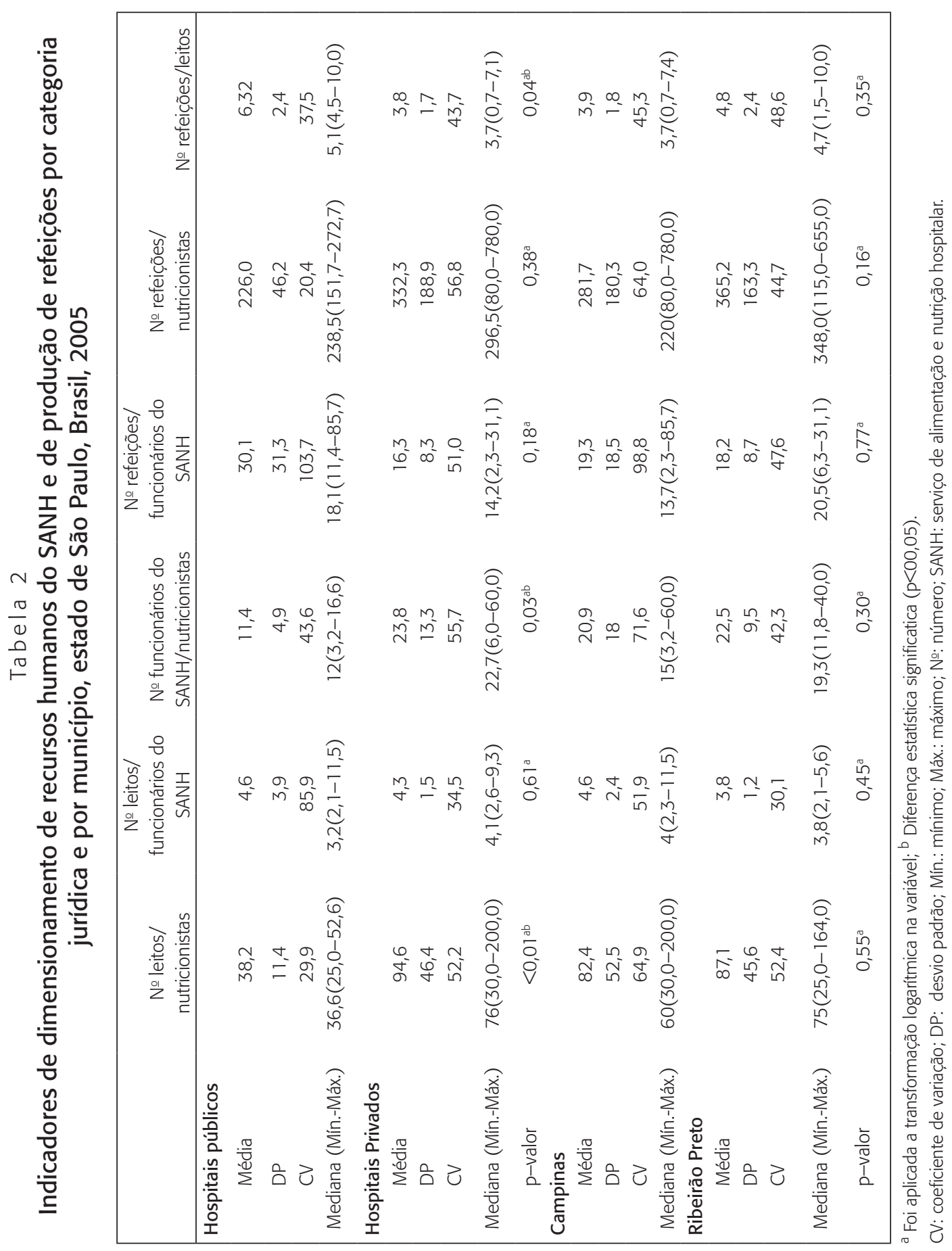




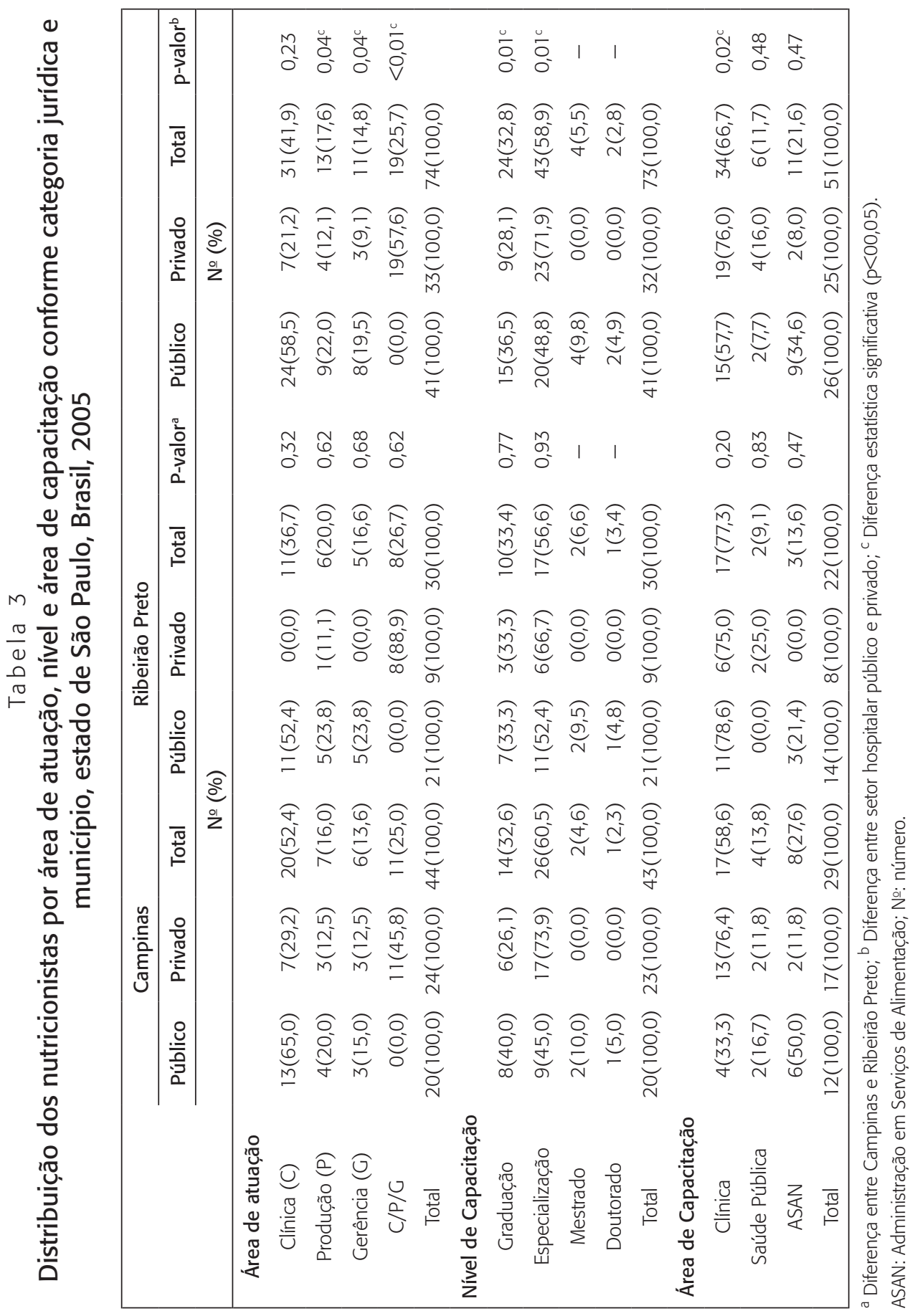


Nos hospitais estudados predominam nutricionistas com mais de 10 anos de formação $(61,6 \%)$. Mais de $2 / 3$ dos nutricionistas $(67,5 \%)$ referiram frequentar cursos de atualização como estratégia para melhorar sua formação profissional. Em Campinas esta proporção foi maior (86,4\%).

A experiência profissional pregressa à inserção no hospital mostra que quase 1/3 $(29,2 \%)$ dos nutricionistas veio da área de produção de refeições em unidades de alimentação e nutrição, a mesma proporção trabalhou anteriormente em outro hospital e 22,2\% não tiveram experiência anterior.

Com relação à infraestrutura no trabalho, $85,2 \%$ das instituições possuíam computador no SANH, e em todos os serviços de hospitais públicos havia este equipamento. Em relação às finalidades do uso do computador, $52,2 \%$ o utilizavam somente para ações na área de produção de refeições. Em $34,8 \%$ das instituições o uso do computador na área de nutrição clínica serve para o cálculo de dietas, orientação de alta hospitalar e outros. A consulta técnica à internet em sites ou em periódicos científicos ainda não é um recurso incorporado aos SANHs.

A jornada de oito horas por dia é o regime de trabalho que predominou em 52\% das instituições. Em Campinas, 58,8\% das instituições apresentam esta jornada de trabalho, e em Ribeirão Preto, $40 \%$ das instituições. A maioria dos profissionais que acumula funções de gerência, clínica e produção $(63,4 \%)$ tem jornada de trabalho de 8 horas ou mais por dia.

O plantão no SANH é realizado em $63 \%$ das instituições hospitalares pesquisadas. Um terço destes hospitais tem plantão na área de nutrição clínica e 58,8\% na área de produção de refeições hospitalares. Em Campinas, apenas $17,6 \%$ dos hospitais têm plantão em nutrição clínica, indicando a priorização da área de produção de refeições.

\section{Discussão}

As diferenças encontradas no dimensionamento de recursos humanos, entre hospitais públicos e privados, em relação ao número de leitos por nutricionistas e número de funcionários do SANH por nutricionista, mostram uma condição mais favorável de profissionais técnicos nos hospitais públicos. Hospitais privados têm mais do que o dobro de leitos e funcionários por nutricionista. Por outro lado, hospitais públicos têm uma média de produção de refeições por leito em torno de 1,5 vez maior, indicando que, apesar da condição de trabalho ser melhor nos hospitais públicos, o aumento da produção de refeições destes hospitais pode absorver mais o tempo de funcionários e nutricionistas. 
Contudo, merece destaque o fato de predominarem hospitais universitários no setor público e apenas $9 \%$ dos hospitais privados serem universitários. De qualquer modo, esta é uma condição da realidade. Entre os hospitais públicos, boa parte tem vínculo com universidades.

A relação do número de funcionários por leito é utilizada como indicador de recursos humanos hospitalares. No Brasil, esses valores variam de 1,0 a 7,2 e em hospitais públicos a variação é de 4,0 a 9,0, podendo nessa variação haver serviços terceirizados que não estão incluídos (Zucchi e Bittar, 2002). Esta relação em países da Organização de Cooperação e Desenvolvimento Econômico (OCDE) variou de 0,7 a 2,6 com relação ao número de funcionários por leito (Escola de Administração da FGV, 2000). Se fosse usada esta mesma relação para os SANHs, teríamos uma média de $0,27(\mathrm{dp} 0,09)$ e mediana de 0,25 funcionário por leito. Ao considerar o número de funcionários do SANH por leito, segundo a natureza jurídica do hospital, temos uma média de 0,25 e mediana 0,24 em SANH de hospitais privados e de 0,32 e 0,32 , respectivamente, em hospitais públicos. Fazer esta análise segmentada por setor hospitalar permitiria comparações e poderia ser utilizada em relação a outras variáveis que indicam a qualidade do serviço.

Zucchi e Bittar (1998) avaliaram a produtividade hospitalar em cinco hospitais de ensino no estado de São Paulo. Verificou-se que o hospital com melhor produtividade foi o que apresentou menor média de permanência, maior índice de renovação, menor intervalo de substituição e a menor relação entre o número de funcionários por leito. Utilizado de forma inversa este indicador, ou seja, número de leitos por funcionário, observaram-se resultados similares na relação de leitos por funcionários nos SANHs de hospitais públicos e privados, porém ambos com um amplo coeficiente de variação. Com relação à produtividade (número de refeições por funcionários), condições muito heterogêneas foram observadas.

O número de leitos por nutricionista deveria variar de acordo com o nível de complexidade do hospital e o nível de atendimento nutricional (Conselho Federal de Nutricionistas, 2005). Para o nível secundário de atendimento nutricional, são recomendados 30 leitos por nutricionista. No nível terciário, mais complexo, esta relação cai pela metade. A maioria dos hospitais estudados ( $82 \%$ eram gerais) tinha demanda para atendimento nutricional secundário e terciário.

O dimensionamento de nutricionistas na área de nutrição hospitalar é diferenciado nos dois segmentos: o de produção de refeições, como um serviço de infraestrutura por fornecer alimentação hospitalar; e o de cuidado nutricional ao paciente hospitalizado, cujas ações são diretamente na enfer- 
maria e têm uma relação mais direta com o número de leitos. Dos 27 hospitais estudados, apenas oito tinham nutricionistas na área de nutrição clínica, 16 tinham apenas um nutricionista e, embora este profissional tenha afirmado dar cobertura na área de nutrição clínica, é questionável dizer que há condições de desenvolvimento de ações simultâneas nestas áreas. A relação de leitos por nutricionistas nesses hospitais foi em média de 98,2 leitos (dp 43,7) e mediana de 90,0. Todos os hospitais com um único nutricionista eram privados. Isso indica que o SANH é reconhecido principalmente pelo fornecimento de refeições, conforme constatado em outros estudos (Garcia, 2006).

Nos hospitais públicos, está mais organizada a distribuição dos nutricionistas nas funções específicas de gerência, produção de refeições e nutrição clínica. Certamente, melhores condições de trabalho podem ser alcançadas quando há especificidade no exercício das ações profissionais. Estudo comparativo entre duas instituições, sobre a situação da gestão dos cuidados nutricionais no setor hospitalar no Brasil e na França, constatou realidades bem diferentes nos dois hospitais estudados. Mesmo com nutricionistas atuando em funções distintas, em ambos os locais foram encontrados fragmentação das tarefas, imprevisibilidade na gestão do processo de produção de refeições (matérias-primas, pessoas e estrutura), pressão temporal para execução de tarefas, gerenciamento de informações de diferentes origens e necessidade de tomar decisões em curto prazo (Souza e Proença, 2004). O acúmulo de funções pode agravar a fragmentação do processo de trabalho, e o próprio deslocamento entre as enfermarias e o setor de produção de refeições inviabiliza a execução plena das funções em ambas as áreas.

Garcia (2006) avaliou as razões que fundamentam a atitude das instituições hospitalares para com a alimentação, a partir da perspectiva dos sujeitos envolvidos, nos diferentes níveis organizacionais. Verificou que na perspectiva dos nutricionistas há dificuldades para melhorar ações voltadas para o paciente devido à falta de sua inserção na equipe multidisciplinar e de reconhecimento de suas ações junto ao paciente, pelas instituições. A própria preocupação com a qualidade sensorial e diversidade de preparações das dietas, segundo estes, deixa a desejar. O estudo retratou a falta de critérios bem estabelecidos e normativos para as funções do nutricionista, sobretudo na atenção nutricional ao paciente hospitalizado.

Estudo sobre as condições de trabalho do nutricionista, realizado no estado do Rio de Janeiro, verificou que 51,3\% dos profissionais atuavam no setor hospitalar, com carga horária semanal de 35,6 horas (Prado e Abreu, 1991). Principalmente nas instituições em que há apenas um nutricionista, o que implica ser o único responsável pelas ações de nutrição, a jornada é de 
oito horas por dia. Outros estudos, nas cidades de São Paulo (Gambardella, Ferreira e Frutuoso, 2000) e de Florianópolis (Vasconcelos, 1991), encontraram resultados semelhantes a este com relação à jornada de trabalho.

A média de nutricionistas com mais de 10 anos de formação, tempo de experiência profissional predominante neste estudo (61,6\%), é de 16,8\% dos nutricionistas da região Sudeste (Akutsu, 2008). Estudo nacional mostra que $1 / 3$ desses profissionais tem esse tempo de formação (Conselho Federal de Nutricionistas, 2006), o que indica que há profissionais com mais tempo de experiência.

No setor público foram encontrados mais nutricionistas com mestrado e doutorado mesmo que em um número reduzido. A capacitação do nutricionista da área hospitalar ainda é incipiente, sobretudo no que diz respeito à pósgraduação stricto sensu, apesar de as cidades estudadas oferecerem diversos programas de pós-graduação na área da saúde. Os dados obtidos são próximos aos encontrados em nível nacional (Conselho Federal de Nutricionistas, 2006) e de outros profissionais da saúde (Martins et al., 2006). Porém, os valores encontrados são inferiores aos do estudo de egressos da Universidade Federal de Santa Catarina (UFSC), em que há 16,8\% de mestres e 9,2\% de doutores (Alves, Rossi e Vasconcelos, 2003), aos 17,2\% de pós-graduados da região Sudeste e aos $12,2 \%$ da região Sul (Akutsu, 2008).

A busca por atualização nesse setor foi alta e revela que o ambiente hospitalar gera demandas para complementar e atualizar o profissional. O conhecimento especializado é importante na estrutura hospitalar de alta complexidade (Souza e Proença, 2004; Farias e Vaitsman, 2002). Nestes serviços a presença de recursos tecnológicos gera a necessidade de profissionais qualificados e o sistema basicamente curativo também aumenta a especificidade do conhecimento. Entre as causas de insatisfação do nutricionista que atua na área hospitalar estão as limitações no exercício de suas competências e a falta de autonomia para a prescrição dietética, que pode ser explicada pela necessidade de melhorar sua capacitação para instrumentá-lo para maior autonomia técnica (Farias e Vaitsman, 2002).

Calabro, Bright e Ball (2001) avaliaram as características da profissão dos nutricionistas em 61 países relacionando-as com o Índice de Desenvolvimento Humano (IDH) e verificaram que, quanto maior o IDH, melhores são as condições da profissão. O Brasil foi classificado com IDH médio, o que mostra ainda a presença de deficiências que se refletem negativamente na formação e na atuação do nutricionista. Estratégias para melhorar a capacitação lato e stricto sensu desses profissionais podem ser uma alternativa para melhorar as ações específicas da área de nutrição hospitalar. 


\section{Conclusão}

Uma ampla heterogeneidade no dimensionamento de recursos humanos do SANH em hospitais públicos e privados foi encontrada. O setor público apresentou uma menor relação de leitos por nutricionista, embora esta relação ainda esteja aquém das recomendações para a área de nutrição hospitalar. Também foi observado um maior número de refeições produzidas por leito, neste segmento, o que pode representar um desvio de recursos humanos a favor do setor de produção de refeições. No setor privado, a priorização do papel do SANH como produtor de refeições é mais acentuada, observada pelo grande número de hospitais onde o nutricionista acumula funções de gerência, produção de refeições e nutrição clínica, condição esta que dificulta o desenvolvimento de ações de cuidado ao paciente.

A necessidade de capacitação do nutricionista da área hospitalar se observa na busca por atualização profissional; contudo, apesar dos municípios estudados serem polos de pós-graduação na área da saúde, a capacitação stricto sensu do nutricionista ainda é sensivelmente inferior aos níveis encontrados nas regiões Sudeste e Sul. Os resultados apontam para a necessidade de se constituírem indicadores de recursos humanos nos SANHs, para o desempenho de ações na área de produção de refeições e de cuidado nutricional ao paciente hospitalizado, particularmente para um delineamento mais preciso do papel do SANH, essencial para melhorar as condições de atenção nutricional hospitalar. Criar mecanismos de capacitação do nutricionista para as áreas de competência da nutrição hospitalar poderia contribuir com aprimoramento do setor. Os indicadores propostos neste estudo precisam ser validados e aprofundados em futuros estudos.

\section{Referências}

AKUTSU, R.C. Os nutricionistas brasileiros: perfil profissional e demográfico. Revista de Nutrição, v. 21, n. 1, p. 7-19, 2008.

ALVES, E.; ROSSI, C.E.; VASCONCELOS, F.A.G. Nutricionistas egressos da Universidade Federal de Santa Catarina: áreas de atuação, distribuição geográfica, índices de pós-graduação e filiação aos órgãos de classe. Revista de Nutrição, v. 16, n. 3, p. 295-304, 2003.

BOSI, M.L.M. Profissionalização e conhecimento: a nutrição em questão. São Paulo: Hucitec, p. 204, 1996. 
CALABRO, K.S.; BRIGHT, K.A.; BALL, S. International perspectives: the profession of dietetics. Nutrition, v. 17, n. 7/8, p. 594-599, 2001.

COELHO-BARROS, E.A. et al. Bayesian analysis for poisson longitudinal data. Revista de Matemática e Estatística, v. 24, n. 3, p. 95-114, 2005.

CONSELHO FEDERAL DE NUTRICIONISTAS. Inserção profissional dos nutricionistas no Brasil. Brasília: Socorro Aquino, 2006.

CONSELHO FEDERAL DE NUTRICIONISTAS. Resolução CFN, n. 380/2005, inciso VIII do artigo $3^{\circ}$ e incisos III, IV, VII e VIII do artigo 4º da Lei n. 8.234, de 17 set. 1991. Atribuição do nutricionista por área de atuação. Brasília. Disponível em: <www. cfn.org.br/novosite/conteudo.aspx?IDMenu=56>. Acesso em: 5 dez. 2007.

ESCOLA DE ADMINISTRAÇÃO DA FUNDAÇÃO GETULIO VARGAS. Núcleo de Pesquisas e Publicações. Relatório de Pesquisa, n. 9, 2000. 157 p. Disponível em: <www. eaesp.fgvsp.br/AppData/GVPesquisa/P00251_1.pdf>. Acesso em: mar. 2009.

FARIAS, L.O.; VAITSMAN, J. Interação e conflito entre categorias profissionais em organizações hospitalares públicas. Cadernos de Saúde Pública, v. 18, n. 5, p. 1229-1241, 2002.

GAMBARDELLA, A.M.D.; FERREIRA, C.F.; FRUTUOSO, M.F.P. Situação profissional de egressos de um curso de nutrição. Revista de Nutrição, v. 9, n. 2, p. 37-40, 2000 .

GARCIA, R.W.D. A dieta hospitalar na perspectiva dos sujeitos envolvidos em sua produção e em seu planejamento. Revista de Nutrição, v. 19, n. 2, p. 129-144, 2006.

LEANDRO-MERHI, V.A. et al. Comparación del estado nutricional, consumo alimentar y tiempo de pacientes de dos hospitales, un publico y otro privado. Nutrición Hospitalaria, v. 21, n. 1, p. 32-37, 2006.

LEANDRO-MERHI, V.A.; GARCIA, R.W.D.; AQUINO, J.L.B. Atención nutricional convencional (ANC) en pacientes quirúrgicos hospitalizados. Nutrición Hospitalaria, v. 22, n. 4, p. 447-454, 2007.

MARTINS, C. et al. Perfil do enfermeiro e necessidades de desenvolvimento de competência profissional. Texto \& Contexto Enfermagem, v. 15, n. 3, p. 472-478, 2006.

MCMAHON, K.; DECKER, G.; OTTERY, F.D. Integrating proactive nutritional assessment in clinical practices to prevent complications and cost. Seminars in Oncology, v. 25, n. 2, Suppl. 6, p. 20-27, 1998.

MINISTÉRIO DA SAÚDE. Cadastro Nacional de Estabelecimentos de Saúde (CNES). 
Disponível em: <http://cnes.datasus.gov.br> . Acesso em: 15 dez. 2007.

MONTGOMERY, D.C. Design and analysis of experiments. 5. ed. New York: John Wiley \& Sons, Inc., 2000.

NAKANO, F.; CAIRASCO, N. Bayesian analysis for poisson longitudinal data. Revista de Matemática e Estatística, v. 24, n. 3, p. 95-114, 2005.

OLIVEIRA, A.F. et al. Evolução nutricional de crianças hospitalizadas e sob acompanhamento nutricional. Revista de Nutrição, v. 18, n. 3, p. 341-348, 2005.

PRADO, S.D.; ABREU, M.S.D. Nutricionista: onde trabalha? Quais suas condições de trabalho? Revista de Nutrição, v. 4, n. 1/2, p. 65-91, 1991.

SAS/STAT ${ }^{\circledR}$ User's Guide, Version 9, Cary, NC. USA: SAS Institute, Inc., 20022003.

SOUZA, A.A.; PROENÇA, R.P.C. Tecnologias de gestão dos cuidados nutricionais: recomendações para qualificação do atendimento nas unidades de alimentação e nutrição hospitalares. Revista de Nutrição, v. 17, n. 4, p. 425-436, 2004.

VASCONCELOS, F.A.G. O nutricionista no Brasil: uma análise histórica. Revista de Nutrição, v. 15, n. 2, p. 127-138, 2002.

VASCONCELOS, F.A.G. Um perfil do nutricionista em Florianópolis-Santa Catarina. Revista Ciências da Saúde, v. 10, n. 1/2, p. 73-86, 1991.

ZUCCHI, P.; BITTAR, O.J.N.V.; HADDAD, N. Produtividade em hospitais de São Paulo, Brasil. Pan American Journal of Public Health/Revista Panamericana de Salud Pública, v. 4, n. 5, p. 311-316, 1998.

ZUCCHI, P.; BITTAR, O.J.N.V. Funcionários por leito: estudo em alguns hospitais públicos e privados. Revista de Administração em Saúde, v. 4, n. 14, p. 1-7, 2002. 
